\title{
Midstream Urine Culture Had a Higher Predictive Value of Post- PNL SIRS in Patients Without Upper Urinary Tract Obstruction
}

\author{
Lizhe An \\ Peking University People's Hospital \\ Liulin Xiong \\ Peking University People's Hospital \\ Qingquan Xu \\ Peking University People's Hospital \\ Xiaobo Huang ( $\nabla$ huang6299@sina.com ) \\ Peking University People's Hospital
}

\section{Research Article}

Keywords: Systemic inflammatory response syndrome, percutaneous nephrolithotomy, MUC, kidney surgery

Posted Date: December 15th, 2021

DOI: https://doi.org/10.21203/rs.3.rs-1062381/v1

License: (c) (i) This work is licensed under a Creative Commons Attribution 4.0 International License. Read Full License 


\section{Abstract \\ Introduction:}

Systemic inflammatory response syndrome (SIRS) is a common complication of percutaneous nephrolithotomy (PNL). Midstream urine culture (MUC) is reported to be associated with postoperative SIRS, but this correlation is uncertain, as MUC may not represent infection of obstructed urine in patients with upper urinary tract obstruction (UUTO). Instead, for patients without UUTO, positive MUC may be an indicator of intrarenal infection. The aim of this study was to investigate whether MUC had a higher predictive value in patients without UUTO.

\section{Materials and Methods}

The data of 175 patients who underwent PNL between September 2015 and June 2016 were retrospectively reviewed. Preoperative and intraoperative factors including MUC were collected and analyzed. The primary endpoint was development of SIRS. Subgroup analysis according to the presence of UUTO was conducted.

\section{Results}

Generally, patients who suffered SIRS had a higher incidence of positive MUC (56\%[25/45] vs. 32\%[41/130], $p=0.005)$. Subgroup analysis showed that among patients without UUTO, positive MUC (75\%[13/17] vs. 33\%[13/39], p=0.003) was still associated with development of SIRS. Among patients with UUTO, however, there was no obvious correlation between positive MUC and SIRS. Multivariate analysis controlling for age, previous ipsilateral kidney surgery, multiple tracts, and blood creatinine conducted in patients without UUTO demonstrated that positive MUC (odds ratio [OR] $=6.419,95 \%$ confidence interval [Cl]: 1.424-28.942, $p=0.016)$ was still associated with development of SIRS. The rate of positive urine culture was $37.7 \%(66 / 72), 72$ isolates of 24 species were reported, including 6 patients with mixed infection and 2 patients with fungi infection. Gram-negative bacteria accounted for a large proportion of all isolates (68.1\%), and Escherichia coli was the most common bacteria (34.7\%).

\section{Conclusions}

MUC had a higher predictive value of post-PNL SIRS in patients without UUTO.

\section{Introduction}

Percutaneous nephrolithotomy (PNL), first described in 1976 by Fernstrom and Johansson [1], has become the standard treatment for lager and complicated renal stones [2]. Infectious complications usually occurred after PNL, specifically, the incidence of fever was $10-30 \%$, systemic inflammatory response syndrome was $35 \%$ and sepsis was $0.9 \%-9.3 \%$ [3-5]. Sepsis rarely occurred after PNL, but it will deteriorate to fetal septic shock with a mortality rate of 50-66\% [6]. Due to its high mortality, judging which patients are susceptible to develop sepsis is necessary. For the close association with sepsis and clear diagnostic criteria, systemic inflammatory response syndrome (SIRS) is a commonly used endpoint to evaluate infection-related complications following PNL [7].

Traditionally, midstream urine culture (MUC) was suggested as a potential factor to predict postoperative infection-related complications and guidance of perioperative antibiotic selection. But several studies indicated that MUC failed to identify patients who develop sepsis post-PNL [8-10]. MUC was shown to have a 15\% positive predictive value for SIRS in one study [10]. A possible explanation for the low predictive value of MUC is midstream urine sample do not accurately represent infection of obstructed urine in patients with upper urinary tract obstruction (UUTO). Instead, for patients without UUTO, positive MUC may be an accurate indicator of intrarenal infection.

The aim of the present study was to test the hypothesis that UUTO may impair the correlation between MUC and SIRS in patients underwent percutaneous nephrolithotomy and to further investigate whether MUC had a higher predictive value in patients without 
UUTO.

\section{Materials And Methods}

After obtaining institutional review board approval, we reviewed all patients who underwent PNL at our institution between September 2015 and June 2016.

Patients' demographics and preoperative variables were collected, including age, gender, presence of diabetes mellitus, placement of ureteral stent, previous ipsilateral kidney surgery. Laboratory tests including midstream urine culture, blood creatinine, blood glucose as well as image examination including ultrasound and CT scan without contrast were performed. Intraoperative variables including operative time, presence of tachycardia, simultaneous ureteroscopy, multiple tracts, size of the tracts, placement of nephrostomy and blood transfusion were collected.

Inclusion criteria were: greater than 18 years of age, underwent PNL, clinical data was complete. Exclusion criteria were: history of malignancy and/or renal failure and/or heart failure, taking immunosuppressant, fever/tachycardia prior to surgery, two-stage PNL.

Previous ipsilateral kidney surgery included previous PNL, ureteroscopy, retrograde intrarenal surgery, partial nephrectomy, pyeloplasty. UUTO was defined as hydronephrosis and/or hydrocalycosis reported by radiologist according to CT scan. A positive midstream urine culture was defined as culture showing growth $>100,000$ colony-forming units (CFUs) of a bacterial species. Operative time was measured as the time from skin incision to placement of nephrostomy or suture. Tachycardia was defined as intraoperative heart rate $>100 / \mathrm{min}$. $24 \mathrm{~F}$ tract was defined as standard tract, compared to $16 \mathrm{~F}$ tract.

All patients received antibiotic prophylaxis $48 \mathrm{~h}$ prior to $\mathrm{PNL}$, this typically involved recommended drug by urine culture or ciprofloxacin $500 \mathrm{mg}$ for patients with negative urine culture.

\section{Endpoint}

The primary endpoint for this study was the development of SIRS in the postoperative hospitalization stay. SIRS was defined as having two of the following: temperature $\geq 38^{\circ} \mathrm{C}$ or $\leq 36^{\circ} \mathrm{C}$, heart rate $>90 /$ minute, respiratory rate $>20 /$ minute, or WBC count $>12,000$ cells $/ \mu \mathrm{L}$ or $<4000$ cells $/ \mu \mathrm{L}$ [11]. At our institution, vitals were measured every 4 hours for the first 24 hours and every 6 hours thereafter.

\section{Statistical analysis}

Statistical analysis was performed using SPSS software (version 19). Mann-Whitney test was used to compare continuous variables, and the Chi square or Fisher's exact tests were used to compare categorical data between groups with and without SIRS. Multivariate analysis was used to study the relationship between MUC and SIRS controlling for other factors. Sensitivity, specificity, positive predictive value (PPV) and negative predictive value (NPV) were used to evaluate the ability of MUC to identify patients at increased risk for developing SIRS. All tests were performed at a significance level of 0.05 .

\section{Results}

The final analysis included 175 patients who met study criteria. Of those patients, 45 (25.7\%) individuals experienced SIRS during the postoperative hospital stay, 119 (68.0\%) patients were combined with UUTO. For patients with UUTO, the incidence of SIRS was $23.5 \%$ (28/119). Among patients without UUTO, the incidence was $30.4 \%$ (17/56) (Table 1). 
Table 1

Demographic data and univariate analysis of risk factors for development of SIRS

\begin{tabular}{|c|c|c|c|c|c|c|c|c|c|}
\hline & \multicolumn{3}{|c|}{ Total $(n=175)$} & \multicolumn{3}{|c|}{ with UUTO ( $n=119)$} & \multicolumn{3}{|c|}{ without UUTO $(n=56)$} \\
\hline & $\begin{array}{l}\text { SIRS+ } \\
(n=45)\end{array}$ & $\begin{array}{l}\text { SIRS- } \\
(n=130)\end{array}$ & $\begin{array}{l}\mathrm{P} \\
\text { value }\end{array}$ & $\begin{array}{l}\text { SIRS+ } \\
(n=28)\end{array}$ & $\begin{array}{l}\text { SIRS- } \\
(n=91)\end{array}$ & $\begin{array}{l}P \\
\text { value }\end{array}$ & $\begin{array}{l}\text { SIRS+ } \\
(n=17)\end{array}$ & $\begin{array}{l}\text { SIRS- } \\
(n=39)\end{array}$ & $\begin{array}{l}\mathrm{P} \\
\text { value }\end{array}$ \\
\hline $\begin{array}{l}\text { Age,median } \\
\text { (IQR),years }\end{array}$ & $47(41-54)$ & $\begin{array}{l}52(42- \\
58.25)\end{array}$ & 0.031 & $\begin{array}{l}44.5(41- \\
50)\end{array}$ & $51(42-58)$ & 0.027 & $51(38-58)$ & $52(39-60)$ & 0.515 \\
\hline Gender,\% (No.) & & & 0.115 & & & 0.186 & & & 0.554 \\
\hline Male & $49 \%(23)$ & $65 \%(84)$ & & $50 \%(14)$ & $65 \%(59)$ & & $53 \%(9)$ & $64 \%(25)$ & \\
\hline Female & $51 \%(22)$ & $35 \%(46)$ & & $50 \%(14)$ & $35 \%(32)$ & & $47 \%(8)$ & $36 \%(14)$ & \\
\hline \multicolumn{10}{|l|}{ Previous history } \\
\hline $\begin{array}{l}\text { Diebetes } \\
\text { Mellitus,\% (No.) }\end{array}$ & $4 \%(2)$ & $16 \%(21)$ & 0.070 & $0 \%(0)$ & $15 \%(14)$ & 0.061 & $12 \%(2)$ & $18 \%(7)$ & 0.854 \\
\hline $\begin{array}{l}\text { Placement of } \\
\text { ureteral stents,\% } \\
\text { (No.) }\end{array}$ & $11 \%(5)$ & $10 \%(13)$ & 1.000 & $14 \%(4)$ & $11 \%(10)$ & 0.890 & $6 \%(1)$ & $8 \%(3)$ & 1.000 \\
\hline $\begin{array}{l}\text { Previous } \\
\text { ipsilateral kidney } \\
\text { surgery,\% (No.) }\end{array}$ & $36 \%(16)$ & $22 \%(29)$ & 0.112 & $43 \%(12)$ & $23 \%(21)$ & 0.041 & $24 \%(4)$ & $21 \%(8)$ & 1.000 \\
\hline \multicolumn{10}{|l|}{$\begin{array}{l}\text { Preoperative } \\
\text { laboratory tests } \\
\text { and image } \\
\text { examination }\end{array}$} \\
\hline UUTO,\% (No.) & $62 \%(28)$ & $70 \%(91)$ & 0.335 & l & / & / & / & / & / \\
\hline $\begin{array}{l}\text { Positive PMUC,\% } \\
\text { (No.) }\end{array}$ & $56 \%(25)$ & $32 \%(41)$ & 0.005 & $43 \%(12)$ & $31 \%(28)$ & 0.259 & $76 \%(13)$ & $33 \%(13)$ & 0.003 \\
\hline $\begin{array}{l}\text { Blood } \\
\text { creatinine,median } \\
\text { (IQR),umol/L }\end{array}$ & $76(66-90)$ & $\begin{array}{l}73.5(62- \\
85)\end{array}$ & 0.344 & $\begin{array}{l}74(58.75- \\
89.75)\end{array}$ & $75(64-87)$ & 0.785 & $\begin{array}{l}78(66- \\
133)\end{array}$ & $69(59-81)$ & 0.032 \\
\hline $\begin{array}{l}\text { Blood } \\
\text { glucose,median } \\
\text { (IQR),mmol/L }\end{array}$ & $\begin{array}{l}5.24(4.845- \\
5.61)\end{array}$ & $\begin{array}{l}5.23(4.82- \\
5.72)\end{array}$ & 0.941 & $\begin{array}{l}5.08(4.81- \\
5.35)\end{array}$ & $\begin{array}{l}5.22(4.83- \\
5.63)\end{array}$ & 0.206 & $\begin{array}{l}5.59(5.21- \\
6.19)\end{array}$ & $\begin{array}{l}5.23(4.80- \\
5.92)\end{array}$ & 0.203 \\
\hline \multicolumn{10}{|l|}{$\begin{array}{l}\text { Intraoperative } \\
\text { factors }\end{array}$} \\
\hline $\begin{array}{l}\text { Operative } \\
\text { time,median } \\
\text { (IQR),min }\end{array}$ & $\begin{array}{l}80(62.5- \\
100)\end{array}$ & $70(50-90)$ & 0.072 & $\begin{array}{l}80(65- \\
100)\end{array}$ & $70(55-95)$ & 0.284 & $\begin{array}{l}80(57.5- \\
102.5)\end{array}$ & $65(50-80)$ & 0.081 \\
\hline $\begin{array}{l}\text { Tachycardia,\% } \\
\text { (No.) }\end{array}$ & $18 \%(8)$ & $9 \%(12)$ & 0.171 & $25 \%(7)$ & $11 \%(10)$ & 0.123 & $6 \%(1)$ & $5 \%(2)$ & 1.000 \\
\hline $\begin{array}{l}\text { Simultaneous } \\
\text { ureteroscopy,\% } \\
\text { (No.) }\end{array}$ & $11 \%(5)$ & $10 \%(13)$ & 1.000 & $18 \%(5)$ & $10 \%(9)$ & 0.419 & $0 \%(0)$ & $10 \%(4)$ & 0.420 \\
\hline $\begin{array}{l}\text { Multiple tracts\% } \\
\text { (No.) }\end{array}$ & $38 \%(17)$ & $22 \%(28)$ & 0.047 & $39 \%(11)$ & $25 \%(23)$ & 0.231 & $35 \%(6)$ & $13 \%(5)$ & 0.114 \\
\hline $24 \mathrm{~F}$ tracts, $\%$ (No.) & $100 \%(45)$ & $96 \%(125)$ & 0.415 & $100 \%(28)$ & $97 \%(88)$ & 1.000 & $100 \%(17)$ & $95 \%(37)$ & 1.000 \\
\hline $\begin{array}{l}\text { Placement of } \\
\text { nephrostomy,\% } \\
\text { (No.) }\end{array}$ & $100 \%(45)$ & $93 \%(121)$ & 0.155 & $100 \%(28)$ & $93 \%(85)$ & 0.368 & $100 \%(17)$ & $93 \%(36)$ & 0.546 \\
\hline
\end{tabular}




\begin{tabular}{|c|c|c|c|c|c|c|c|c|c|}
\hline \multicolumn{4}{|c|}{ Total $(n=175)$} & \multicolumn{3}{|c|}{ with UUTO ( $n=119)$} & \multicolumn{3}{|c|}{ without UUTO $(n=56)$} \\
\hline $\begin{array}{l}\text { Blood } \\
\text { transfusion,\% } \\
\text { (No.) }\end{array}$ & $4 \%(2)$ & $2 \%(2)$ & 0.585 & $7 \%(2)$ & $1 \%(1)$ & 0.138 & $0 \%(0)$ & $3 \%(1)$ & 1.000 \\
\hline
\end{tabular}

Generally, patients who suffered SIRS had a higher incidence of positive MUC (56\%[25/45] vs. 32\%[41/130], $p=0.005)$, were younger than the other patients (47[41-54] vs. 52[42-58.25], $\mathrm{p}=0.031)$, and were more likely to received multiple tracts surgery (38\% [17/45] vs. $22 \%[28 / 130], p=0.047)$. Other factors including gender, previous history, preoperative laboratory tests, operative time and blood transfusion showed no difference between groups $(p>0.05)$ (Table 1$)$.

Among patients with UUTO, the incidence of positive MUC was similar between patients with and without SIRS (43\%[12/28] vs. $31 \%[28 / 91], p=0.259)$. Meanwhile, younger age (44.5[41-50] vs. 51[42-58], $p=0.027)$ and previous ipsilateral kidney surgery (43\% [12/28] vs. 23\%[21/91], $p=0.041$ ) were associated with development of SIRS (Table 1).

Among patients without UUTO, positive MUC (76\%[13/17] vs. 33\%[13/39], $p=0.003)$ as well as elevation of blood creatinine (78[66133] vs. 69[59-81], p=0.032) were associated with development of SIRS. Unlike the entire sample, there was no difference could be seen in age $(p>0.05)($ Table 1$)$.

Positive MUC had sensitivity, specificity, positive predictive value (PPV) and negative predictive value (NPV) of 55.6\%, 68.5\%, 37.9\%, and $81.7 \%$, respectively, for development of SIRS of all patients.

When the effect of UUTO was excluded, among patients without UUTO, the sensitivity increased to $76.5 \%$, specificity remained similar at $66.7 \%$, PPV increased to $50.0 \%$, and NPV increased to $86.7 \%$.

Multivariate analysis was conducted to test stability of the relationship between MUC and development of SIRS among patients without UUTO, controlling factors including age, previous ipsilateral kidney surgery, multiple tracts and blood creatinine. Result showed that positive MUC (odds ratio [OR] = 6.419, 95\% confidence interval [CI]: 1.424-28.942, p=0.016) was still associated with development of SIRS (Table 2).

Table 2

Multivariate analysis of risk factors for development of SIRS among patients without UUTO

\begin{tabular}{|c|c|c|c|c|c|c|c|}
\hline \multirow[t]{2}{*}{ Variables } & \multirow[t]{2}{*}{$\beta$ value } & \multirow[t]{2}{*}{ S.E, } & \multirow[t]{2}{*}{ Wals } & \multirow[t]{2}{*}{$P$ value } & \multirow[t]{2}{*}{$O R$} & \multicolumn{2}{|c|}{ 95\% C.I. } \\
\hline & & & & & & Lower & Upper \\
\hline Age,years & -.015 & .028 & .290 & .590 & .985 & .932 & 1.041 \\
\hline Positive PMUC & 1.859 & .768 & 5.855 & .016 & 6.419 & 1.424 & 28.942 \\
\hline Previous ipsilateral kidney surgery & -.923 & 1.004 & .845 & .358 & .397 & .055 & 2.844 \\
\hline Multiple tracts & 1.160 & .901 & 1.657 & .198 & 3.189 & .546 & 18.645 \\
\hline Blood creatinine & .031 & .014 & 4.934 & .026 & 1.031 & 1.004 & 1.059 \\
\hline Constant & -3.723 & 1.896 & 3.854 & .050 & .024 & & \\
\hline
\end{tabular}

Among 175 patients included in this study, 66(37.7\%) patients had positive urine culture, yielding 72 isolates of 24 species. Mixed infection (two kinds of bacteria) appeared in 6 patients, including three patients infected with Escherichia coli and Enterococcus faecalis, one patient infected with Escherichia coli and Stenotrophomonas maltophilia, one patient infected with Escherichia coli and Klebsiella pneumoniae, and one patient infected with Enterococcus faecalis and Citontobacter bureli. Gram-negative bacteria accounted for a large proportion of all isolates, with the percent of $68.1 \%(49 / 72)$. Escherichia coli was the most common bacteria (34.7\%), followed by Proteus mirabilis (13.9\%), Enterococcus faecalis (11.1\%), Pseudomonas aeruginosa (4.2\%), Klebsiella pneumoniae (4.2\%), Streptococcus agalactiae (2.8\%), Staphylococcus haemolyticus $(2.8 \%)$, Streptococcus mitis $(2.8 \%)$ and Staphylococcus epidermidis (2.8\%) (Figure 1). It should be noted that fungal infection occurred in two patients (3.0\%), including 
one 49-year-old male patient infected with Candida parapsilosis and one 27-year-old female patients infected with Candida glabrata, and both of them developed SIRS postoperatively.

\section{Discussion}

Infectious complication usually emerges after PCNL, which could deteriorate to fatal urosepsis. Several studies have reported preoperative and intraoperative risk factors for the development of SIRS following PNL, including stone burden, hydronephrosis, staghorn stone, multiple tracts, longer operative time, the presence of purulent substance obtained on puncture, positive pelvic urine culture, positive stone culture and so on $[12,13]$.

Pathogenic microorganisms colonized in urinary tract are source of infection. So, urologists emphasize the importance of bacterial culture. Due to convenience and easiness to collect, urine culture is routinely performed before PNL to ensure sterility, and appropriate antibiotics were used for patients with infection, but urosepsis is still a serious complication in patients undergoing PNL $[10,14]$. Comparing to higher predictive value of stone culture and pelvic urine culture [15], midstream urine culture is reported as a poor predictor for development of SIRS following lithotripsy [8-10], one study by Dogan et al [16] showed that $66 \%$ of 82 patients with SIRS had negative urine culture.

This poor predictive value of midstream urine culture could possibly be explained that midstream urine sample do not accurately represent infection of obstructed urine in patients with upper urinary tract obstruction. Felix Cheung et al. [17] reported that Bacteria on urine microscopy was not associated with other markers of systemic infection and may largely represent a contaminant. Similar conclusion was also made by Paramananthan Mariappan et al. [18], they found that in obstructive uropathy secondary to a stone midstream urine culture was a poor predictor of infected urine proximal to the obstruction and infected stones. But no research has investigated whether midstream urine culture could predict postoperative infectious complications more accurately in the absence of UUTO.

The results of our study verified the hypothesis that UUTO impaired the correlation between MUC and SIRS pro and coin. On the one hand, positive MUC had a higher predictive value without the effect of UUTO, on the other hand, positive MUC was not associated with development of SIRS among patients with UUTO. Multivariate analysis controlling for other related factors showed that UUTO still correlated with the development of SIRS after PNL.

Our study brought several reported risk factors into analysis, the result showed that positive MUC, multiple tracts, ipsilateral kidney surgery, and blood creatinine were associated with development of SIRS, which was in accord with previous studies [12, 19,20]. We also found that patients suffered SIRS was younger than the other patients, narrow age range of patients may account for this result, and some studies also reported that age was not a significant factor [21, 22].

Escherichia coli was reported as the most common bacteria associated with urinary tract infection, with the prevalence of 50.0\%-82.8\% [23-26]. But for patients with stones, data form China reported relative lower prevalence of Escherichia coli, which ranged from 29.3-48.7\% [27-29], and was in accord with our results. This discrepancy may be explained by difference of bacteria distribution in different population and regions.

There are several limitations of our study. UUTO was defined as hydronephrosis and/or hydrocalycosis in this study, this was a major confounding factor, because in cases with hydrocalycosis pelvic urine could flow to bladder, so MUC could partly represent upper urinary tract infection. Second, this was a retrospective study, only data were important enough to be recorded could be analyzed. Third, the effects of other potential covariates on developing SIRS after PNL such as, prophylactic antibiotic administration, previous history of urinary traction infection, stone burden, and irritation pressure were not taken into account in this study.

\section{Conclusions}

Upper urinary tract obstruction could impair the relationship between preoperative midstream urine culture and post-PNL SIRS. Midstream urine culture had a higher predictive value of post-PNL SIRS in patients without upper urinary tract obstruction. 


\section{Declarations}

\section{Ethics approval and consent to participate}

The study was conducted in accordance with the Declaration of Helsinki (as revised in 2013). The study was approved by the ethics board of Peking University People's Hospital Review Board and all patients signed the preoperative informed consent.

\section{Consent for publication}

Not applicable.

\section{Availability of data and materials}

The datasets used and analyzed during the current study are available from the corresponding author on reasonable request.

\section{Competing interests}

The authors have no conflicts of interest to declare.

\section{Funding}

Not applicable.

\section{Authors' contributions}

L.A. collected the data and wrote the manuscript. L.X. edited the manuscript. Q.X. managed the data. X.H. developed the project. All authors reviewed the manuscript.

\section{Acknowledgement}

Not applicable.

\section{References}

1. Fernstrom I, Johansson B. Percutaneous pyelolithotomy. A new extraction technique. Scand J Urol Nephrol. 1976 1976-01-19: 10:257-9

2. de la Rosette J, Assimos D, Desai M, et al. The Clinical Research Office of the Endourological Society Percutaneous Nephrolithotomy Global Study: indications, complications, and outcomes in 5803 patients. J ENDOUROL. 2011 2011-01-01: 25:11-7

3. Michel MS, Trojan L, Rassweiler JJ. Complications in percutaneous nephrolithotomy. EUR UROL. 2007 2007-04-01: 51:899906, 906

4. Draga RO, Kok ET, Sorel MR, Bosch RJ, Lock TM. Percutaneous nephrolithotomy: factors associated with fever after the first postoperative day and systemic inflammatory response syndrome. J ENDOUROL. 2009 2009-06-01: 23:921-7

5. Margel D, Ehrlich Y, Brown N, Lask D, Livne PM, Lifshitz DA. Clinical implication of routine stone culture in percutaneous nephrolithotomy-a prospective study. UROLOGY. 2006 2006-01-01: 67:26-9

6. Singh P, Yadav S, Singh A, et al. Systemic Inflammatory Response Syndrome Following Percutaneous Nephrolithotomy: Assessment of Risk Factors and Their Impact on Patient Outcomes. UROL INT. 2016 2016-01-20: 96:207-11

7. Rhodes A, Evans LE, Alhazzani W, et al. Surviving Sepsis Campaign: International Guidelines for Management of Sepsis and Septic Shock: 2016. Intensive Care Med. 2017 2017-03-01: 43:304-77

8. Eswara JR, Shariftabrizi A, Sacco D. Positive stone culture is associated with a higher rate of sepsis after endourological procedures. UROLITHIASIS. 2013 2013-10-01: 41:411-4

9. Gonen M, Turan H, Ozturk B, Ozkardes H. Factors affecting fever following percutaneous nephrolithotomy: a prospective clinical study. J ENDOUROL. 2008 2008-09-01: 22:2135-8 
10. Mariappan P, Smith G, Bariol SV, Moussa SA, Tolley DA. Stone and pelvic urine culture and sensitivity are better than bladder urine as predictors of urosepsis following percutaneous nephrolithotomy: a prospective clinical study. J Urol. 2005 2005-05-01: 173:1610-4

11. Bone RC, Balk RA, Cerra FB, et al. Definitions for sepsis and organ failure and guidelines for the use of innovative therapies in sepsis. The ACCP/SCCM Consensus Conference Committee. American College of Chest Physicians/Society of Critical Care Medicine. CHEST. 1992 1992-06-01: 101:1644-55

12. Chen L, Xu QQ, Li JX, Xiong LL, Wang XF, Huang XB. Systemic inflammatory response syndrome after percutaneous nephrolithotomy: an assessment of risk factors. INT J UROL. 2008 2008-12-01: 15:1025-8

13. Singh P, Yadav S, Singh A, et al. Systemic Inflammatory Response Syndrome Following Percutaneous Nephrolithotomy: Assessment of Risk Factors and Their Impact on Patient Outcomes. UROL INT. 2016 2016-01-20: 96:207-11

14. Wolf JJ, Bennett CJ, Dmochowski RR, Hollenbeck BK, Pearle MS, Schaeffer AJ. Best practice policy statement on urologic surgery antimicrobial prophylaxis. J Urol. 2008 2008-04-01: 179:1379-90

15. Roushani A, Falahatkar S, Sharifi SH, et al. Intra-operative stone culture as an independent predictor of systemic inflammatory response syndrome after percutaneous nephrolithotomy. UROLITHIASIS. 2014 2014-10-01: 42:455-9

16. Dogan HS, Guliyev F, Cetinkaya YS, Sofikerim M, Ozden E, Sahin A. Importance of microbiological evaluation in management of infectious complications following percutaneous nephrolithotomy. INT UROL NEPHROL. 2007 2007-01-20: 39:737-42

17. Cheung F, Loeb CA, Croglio MP, Waltzer WC, Weissbart SJ. Bacteria on Urine Microscopy Is Not Associated with Systemic Infection in Patients with Obstructing Urolithiasis. J ENDOUROL. 2017 2017-09-01: 31:942-5

18. Mariappan P, Loong CW. Midstream urine culture and sensitivity test is a poor predictor of infected urine proximal to the obstructing ureteral stone or infected stones: a prospective clinical study. J Urol. 2004 2004-06-01: 171:2142-5

19. Kumar S, Bag S, Ganesamoni R, Mandal AK, Taneja N, Singh SK. Risk factors for urosepsis following percutaneous nephrolithotomy: role of 1 week of nitrofurantoin in reducing the risk of urosepsis. Urol Res. 2012 2012-02-01: 40:79-86

20. Draga RO, Kok ET, Sorel MR, Bosch RJ, Lock TM. Percutaneous nephrolithotomy: factors associated with fever after the first postoperative day and systemic inflammatory response syndrome. J ENDOUROL. 2009 2009-06-01: 23:921-7

21. He Z, Tang F, Lei H, Chen Y, Zeng G. Risk factors for systemic inflammatory response syndrome after percutaneous nephrolithotomy. PROG UROL. 2018 2018-10-01: 28:582-7

22. Rivera M, Viers B, Cockerill P, Agarwal D, Mehta R, Krambeck A. Pre-and Postoperative Predictors of Infection-Related Complications in Patients Undergoing Percutaneous Nephrolithotomy. J ENDOUROL. 2016 2016-09-01: 30:982-6

23. Hisano M, Bruschini H, Nicodemo AC, Gomes CM, Lucon M, Srougi M. The Bacterial Spectrum and Antimicrobial Susceptibility in Female Recurrent Urinary Tract Infection: How Different They Are From Sporadic Single Episodes? UROLOGY. 2015 2015-0901: 86:492-7

24. Paranjpe I, Kapoor A, Tran T, et al. Multi-Institutional Predictors of Antibiotic Resistance in Patients Presenting to the Emergency Department with Urosepsis Secondary to Ureteral Obstruction. J ENDOUROL. 2021 2021-01-01: 35:97-101

25. Kornfalt IH, Melander E, Hedin K, Molstad S, Beckman A. Uncomplicated urinary tract infections in Swedish primary care; etiology, resistance and treatment. BMC INFECT DIS. 2019 2019-02-13: 19:155

26. Rossignol L, Vaux S, Maugat S, et al. Incidence of urinary tract infections and antibiotic resistance in the outpatient setting: a cross-sectional study. INFECTION. 2017 2017-02-01: 45:33-40

27. Chen D, Zhang $Y$, Huang J, et al. The analysis of microbial spectrum and antibiotic resistance of uropathogens isolated from patients with urinary stones. INT J CLIN PRACT. 2018 2018-06-01: 72:e13205

28. Wang S, Zhang Y, Zhang X, Li J. An evaluation of multidrug-resistant (MDR) bacteria in patients with urinary stone disease: data from a high-volume stone management center. WORLD J UROL. 2020 2020-02-01: 38:425-32

29. Wang S, Shi YK, Huang XB, et al. [Bacterial culture and drug sensitivity analysis of upper urinary tract calculi complicating with infection]. Beijing Da Xue Xue Bao Yi Xue Ban. 2014 2014-10-18: 46:798-801

\section{Figures}




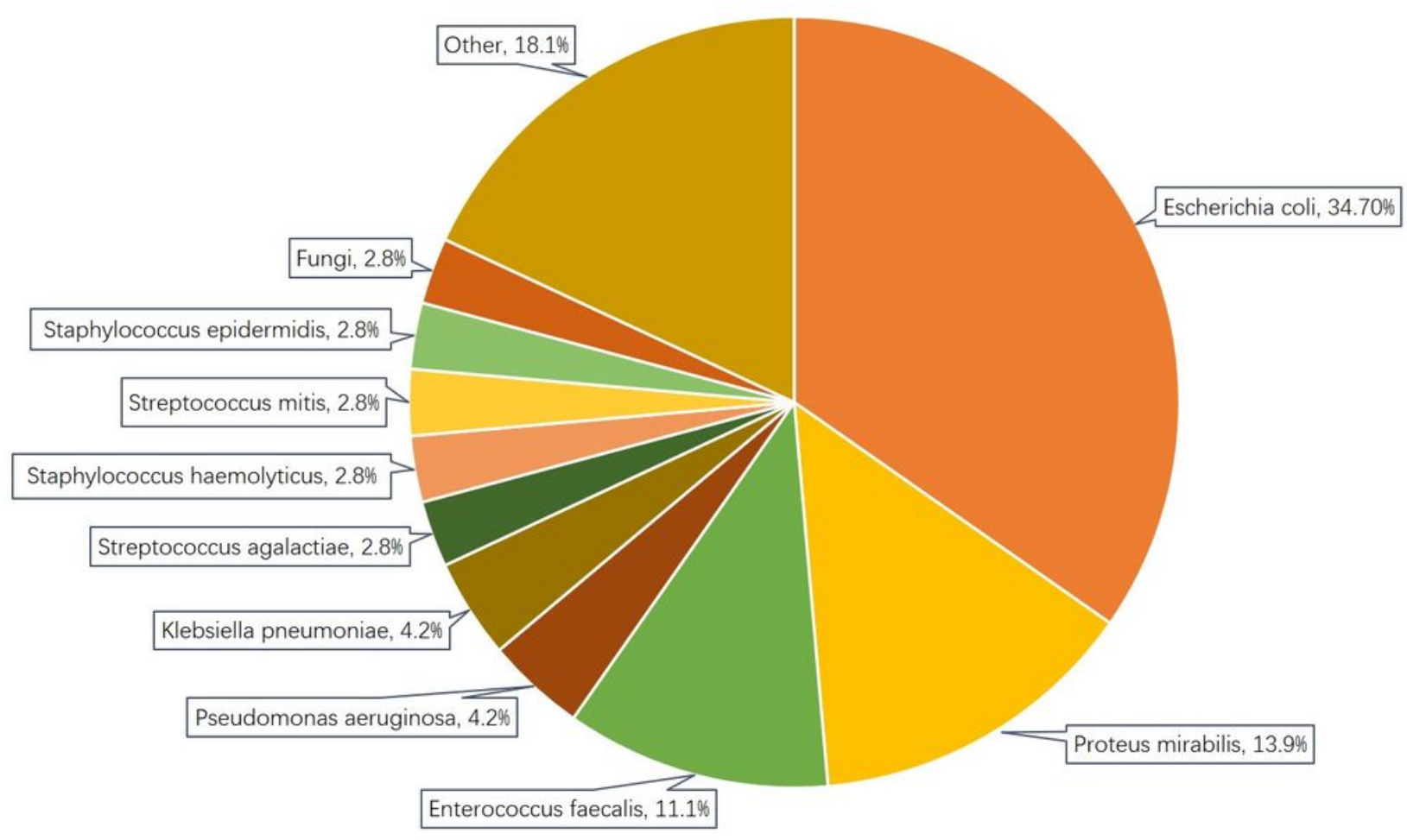

\section{Figure 1}

Among 175 patients included in this study, 66(37.7\%) patients had positive urine culture, yielding 72 isolates of 24 species. Mixed infection (two kinds of bacteria) appeared in 6 patients, including three patients infected with Escherichia coli and Enterococcus faecalis, one patient infected with Escherichia coli and Stenotrophomonas maltophilia, one patient infected with Escherichia coli and Klebsiella pneumoniae, and one patient infected with Enterococcus faecalis and Citontobacter bureli. Gram-negative bacteria accounted for a large proportion of all isolates, with the percent of $68.1 \%(49 / 72)$. Escherichia coli was the most common bacteria (34.7\%), followed by Proteus mirabilis (13.9\%), Enterococcus faecalis (11.1\%), Pseudomonas aeruginosa (4.2\%), Klebsiella pneumoniae (4.2\%), Streptococcus agalactiae (2.8\%), Staphylococcus haemolyticus (2.8\%), Streptococcus mitis $(2.8 \%)$ and Staphylococcus epidermidis $(2.8 \%)$ 